\title{
A 32-GHz Reflected-Wave Maser Amplifier With Wide Instantaneous Bandwidth
}

\author{
J. Shell and D. Neff \\ Radio Frequency and Microwave Subsystems Section
}

\begin{abstract}
An eight-stage, 32-GHz reflected-wave ruby maser has been built. The maser operates in a 3-watt closed-cycle refrigerator (CCR) at $4.5 \mathrm{~K}$ and is capable of $21 \mathrm{~dB}$ of net gain with an instantaneous bandwidth of $400 \mathrm{MHz}$. The input noise temperature referred to the room-temperature flange is approximately $21 \mathrm{~K}$.
\end{abstract}

\section{Introduction}

In recent years, NASA has been evaluating the relative advantages of $8.4-\mathrm{GHz}, 32 \cdot \mathrm{GHz}$, and optical communication links for its future deep space communication needs [1]. As a result, the Deep Space Network is planning to upgrade its current capabilities by the addition of a $32 \cdot \mathrm{GHz}$ downlink [2]. The $500-\mathrm{MHz}$ band, from 31.8 to $32.3 \mathrm{GHz}$, is allocated for that purpose [3]. An important component in achieving that goal is the development of low-noise front-end amplifiers. One promising approach, drawing upon JPL's experience and success with masers, is to develop a $32-\mathrm{GHz}$ ruby maser.

This article describes the first such device built at $32 \mathrm{GHz}$ by the Cryo-Electronics Group at JPL. The design of this maser draws upon previous experience in building reflectedwave amplifiers at $22 \mathrm{GHz}$ (K-band) [4]. The reflected-wave maser (a negative resistance amplifier utilizing a shorted ruby-filled transmission line) design is considered a more realizable approach for broadband $32-\mathrm{GHz}$ performance than the traveling-wave maser (utilizing a periodic transmission line with distributed isolators) currently in use at 2 and $8 \mathrm{GHz}$.
This maser will initially be part of a receiver system for the Caltech Owens Valley Radio Observatory (OVRO) located near Big Pine, California. Provision has been made to accommodate either the 5.5-meter Cassegrain antenna with high-gain feedhorns or the 40-meter antenna with focal point feeds. Caltech personnel will operate and maintain the equipment for 32- $\mathrm{GHz}$ radio astronomy, specifically for use in measuring the microwave cosmic background (approximately $3 \mathrm{~K}$ ). The benefit to JPL/NASA will derive from the receipt of weather data for the 32-GHz communications band, from the calibration of known radio stars, and from the acquisition of operational data on the maser's performance and experience in running the maser as part of a system.

\section{Input Description}

The design (see Figs. 1 and 2) uses two input feedhorns spaced 4.5 inches apart that will be symmetrically placed about the center line of the 5.5-meter antenna and asymmetrically placed about the center line of the 40-meter antenna. The 
input lines are identical in structure, and each consists of the following:

(1) A mica window.

(2) A vacuum feedthrough waveguide.

(3) A cryogenically coolable low-noise choked waveguide, nominally ambient to $4.5 \mathrm{~K} .^{1}$

(4) A round-to-square waveguide adapter $(0.343$ round to 0.234 square).

(5) A polarizer with RCP and LCP outputs. ${ }^{2}$

(6) A switching circulator to direct inputs to the maser from either the antenna or a variable-temperature cold load. ${ }^{3}$

(7) A 30-dB cross-guide directional coupler for external signal calibration and noise diode inputs.

A third EMS switching circulator switches the maser input between the two antenna input lines at any rate up to $5 \mathrm{kHz}$. The signal input line is finally connected to the maser circulator block. (Operation of the maser will be fully described in the following sections.)

\section{Maser Description}

A block diagram of the maser is shown in Fig. 3. It consists of eight stages of ruby-filled waveguide connected in series with 18 junction circulators. This design was chosen to achieve our goal of low noise and wide instantaneous bandwidth. Low noise is possible because of lower dissipative losses than those typically achieved in a traveling-wave maser with slow-wave structures and isolators. The reflected-wave maser uses only the dielectric and waveguide slowing of the ruby-filled guide. Wide instantaneous bandwidth is achieved by using a long length of ruby (approximately $67 \mathrm{~cm}$ in total length) and artificially broadening the ruby linewidth with a magnetic field gradient.

The number of stages and the length of each stage are dictated by our goal of low-gain ripple, convenient magnet size, ease of fabrication of the ruby bars, and good noise performance across the band. The absence of a resonant slow-wave structure also allows for relatively large tunability. The tuning range is limited only by the frequency response of the junction circulators. This particular maser will tune from approximately

\footnotetext{
${ }^{1}$ Modified version of U.S. Patent $\# 4,215,327$.

${ }^{2}$ Model 2800, Atlantic Microwave Corporation, Boston, Massachusetts.

${ }^{3}$ Model 408-26, Electromagnetics Sciences, Inc. (EMS), Norcross, Georgia.
}

29 to $34 \mathrm{GHz}$. However, because of the large pump power required to invert the large volume of ruby, we have chosen to operate with fixed tuned "pump" oscillators, effectively making this a fixed-frequency amplifier at this time.

Pump power requirements are more stringent for the 32 $\mathrm{GHz}$ masers than for the $22-\mathrm{GHz}$ masers. Waveguide and structure losses are higher, and the ruby is more difficult to invert due to smaller pump transition probabilities. More importantly, the pump power is divided eight ways rather than four ways as in the $22-\mathrm{GHz}$ maser. The power is supplied by four independent oscillators. It is combined in pairs with two $3-\mathrm{dB}$ hybrid couplers ${ }^{4}$ outside the vacuum vessel. The four waveguide outputs from the hybrids enter the closed-cycle refrigerator (CCR) and are split with four E-plane power splitters into eight channels. ${ }^{5}$ The power is then carried to each ruby separately in WR 15 waveguide and impedance-matched to the ruby-filled guide. This ensures an even power distribution among all eight stages.

Almost all of the unfilled signal waveguide is a specially fabricated waveguide. ${ }^{6}$ The waveguide width for $32 \mathrm{GHz}$ was chosen by frequency scaling the WR42 waveguide of the K-band maser from its best operating frequency of $23.3 \mathrm{GHz}$. The height was chosen to obtain broader-bandwidth circulator operation and to ease the matching into the ruby-filled guide.

The signal enters the circulator block and passes through two junctions. The first junction, configured as an isolator with a termination on the third port, reduces the level of reflected signals exiting the input. This function is performed by all the terminated junctions. After passing through the input isolator, the signal enters the input circulator and then the first ruby bar. The signal travels the full length of the ruby bar, growing exponentially with distance, and is reflected at the opposite end. It travels back up the bar, still being amplified, and is directed into the second stage. The gain of each stage depends on the chosen bandwidth. The signal makes a total of 26 passes through the junctions and 16 passes through the ruby. The forward insertion loss (at $4.5 \mathrm{~K}$ ) is approximately $12 \mathrm{~dB}$.

The ruby is single crystal and is grown by the Czochralski process by Union Carbide. The chromium-to-aluminum con-

\footnotetext{
${ }^{4}$ Model 15HS1 2B, Microwave Development Laboratories, Inc., Natick, Massachusetts.

${ }^{5}$ The power splitters were manufactured at JPL. A commercial source is not known to the authors.

${ }^{6}$ Copper (OFHC) waveguide $(0.051$ inch by 0.306 inch), A. T. Wall Company, Warwick, Rhode Island.
} 
centration ratio is approximately 0.05 percent. The ruby is cut and oriented so that the optic-axis is at an angle of 54.73 degrees to the $\mathrm{dc}$ magnetic field. The energy levels of the ground state orbital of the chromium spins are shown in Fig. 4 as a function of magnetic field for this orientation. Population inversion is achieved by push-pull pumping the $1-3$ and the $2-4$ transitions [5]. The signal transition is the $2-3$ transition.

\section{A. Maser Gain and Bandwidth}

The natural linewidth of ruby is $60 \mathrm{MHz}$. In a maser with a uniform magnetic field and $30 \mathrm{~dB}$ of electronic gain, the 3-dB bandwidth is $20 \mathrm{MHz}$, about one-third of the linewidth. Therefore, to achieve masers with wider instantaneous bandwidths, it is common practice to inhomogeneously broaden the line shape by placing different parts of the ruby in different dc magnetic fields [5]. For large bandwidths, an efficient and convenient technique is to employ a linearly staggered magnetic field along the length of the ruby. This is the technique employed here. The frequency dependence of the gain is given by $[5]$

$$
\begin{aligned}
G(\nu)= & \frac{g}{2} \frac{w}{d \nu}\left\{\tan ^{-1}\left[\frac{2\left(\nu-\nu_{0}\right)}{w}+\frac{d \nu}{w}\right]\right. \\
& \left.-\tan ^{-1}\left[\frac{2\left(\nu-\nu_{0}\right)}{w}-\frac{d \nu}{w}\right]\right\}
\end{aligned}
$$

where

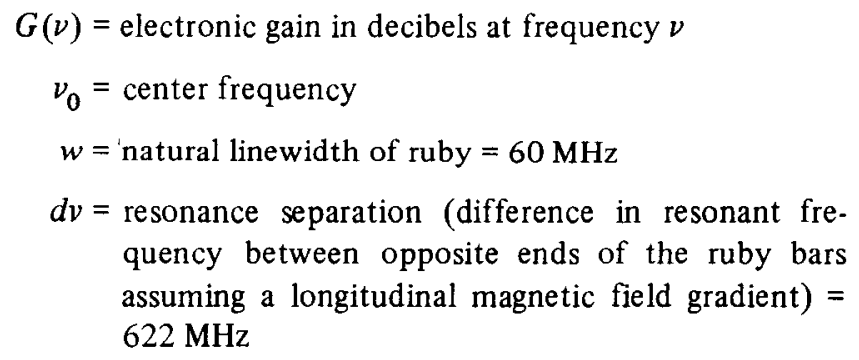

and $g$, the unbroadened gain (gain without artificial broadening), is given by

$$
g=\frac{27 S N}{Q_{m}}
$$

where, in turn,

$$
\begin{aligned}
S & =\text { slowing factor }=\left(c / V_{\text {group }}\right)=3.9 \\
N & =\text { length of ruby in free space wavelengths }=143 \\
Q_{m} & =\text { magnetic } Q
\end{aligned}
$$

and

$$
\begin{aligned}
\frac{1}{Q_{m}} & =\frac{\gamma^{2} h}{\pi \mu_{0}}\left(\frac{h \nu}{k T}\right) \frac{N}{n} \frac{I \sigma^{2} \eta}{w} \\
& =1.38\left(10^{-18}\right) \frac{h \nu}{k T} \frac{N}{n} \frac{I \sigma^{2} \eta}{w}
\end{aligned}
$$

where

$$
\begin{aligned}
\gamma= & \text { magnetogyric ratio }=2.8 \mathrm{MHz} / \text { gauss } \\
h= & \text { Planck's constant } / 2 \pi=1.054 \mathrm{E}-27 \text { erg-sec } \\
I= & \text { inversion ratio (gain with pumps on } / \text { absorption with } \\
& \text { pumps off) }=0.7 \\
\nu= & \text { frequency in hertz } \\
T= & \text { absolute temperature }=4.5 \mathrm{~K} \\
k= & \text { Boltzmann's constant } \\
N= & \text { spin density per cubic centimeter }=2.35 \mathrm{E} 19 \\
n= & \text { number of energy levels }=4 \\
\sigma^{2}= & \text { signal transition probability }=0.96 \\
\eta= & \text { filling factor }=0.5 \\
\mu_{0}= & \text { permeability of free space } \\
w= & 60 \mathrm{MHz}=\text { natural linewidth of ruby in units of mega- } \\
& \text { hertz }
\end{aligned}
$$

In this instance, $1 / Q_{m}=2.29 \mathrm{E}-02$. Therefore $g=232 \mathrm{~dB}$. Assuming a resonance separation of $622 \mathrm{MHz}$ (corresponding to our tapered shim), the bandpass of the maser looks as shown in Fig. 5. This is the electronic gain (and does not include the maser structure insertion loss) and agrees quite well with what is observed. Obviously, gain can be traded for bandwidth and vice versa.

\section{B. Noise Temperature}

The noise temperature of a reflected-wave maser can be thought of as arising from two distinct noise sources. The first source is blackbody radiation from any dissipative losses in the system. The second source is spontaneous emission from the ruby. The theoretical noise temperature of the maser referred to the room temperature flange can be calculated if estimates of the losses and the state of the ruby are known. The results of these estimates are plotted in Fig. 6 and tabulated in Table 1. The noise temperature is a function of gain (and therefore bandwidth) for two reasons. First, as the gain per stage decreases (with increasing bandwidth), the ratio of loss per unit length to gain per unit length increases because the losses 
are constant. This causes a large increase in noise temperature, especially for values of this ratio greater than $0.5[6]$. Second, as the gain per stage is decreased, noise from succeeding ruby stages makes a larger contribution to the total noise temperature. (The noise contribution of a given stage, when referred to the input of the complete amplifier, is given by the equivalent noise contribution at the input of the stage divided by the net gain which precedes that stage.)

\section{Gain Ripple}

There are several sources of gain ripple in this maser. One source is mismatch between the circulator junctions and the rubies. Another source is cross-talk between ruby stages where the structure mates with connecting waveguide flanges. A third is uneven pumping of the rubies due to a frequency-dependent coupling of pump power. Of these different sources of ripple, only the first is amenable to calculation. The VSWR of reduced-height guide to ruby-filled guide has been measured at room temperature and is typically 1.21 . This is determined by measuring with a reflectometer the attenuation and ripple with the waveguide shorted at the pump end. ${ }^{7}$ The VSWR into the circulator junctions has also been measured at room temperature and is typically 1.32 . This was measured by placing the entire circulator block in a 12-inch Varian electromagnet. Therefore, the equivalent circulator-ruby VSWR is somewhere between 1.09 and 1.60 , depending on the frequency and path length between them.

\section{Maser Structure}

The copper structure that houses the eight ruby bars is constructed in three parts in a sandwich configuration (see Fig. 7). The center section is flat on both sides and forms one broad wall of the waveguide. The remaining three walls are formed by machining waveguide channels in the upper and lower sections of the sandwich. The dimensions of the ruby-filled guide are 0.100 by $0.050 \mathrm{inch}$. There are four waveguide channels in each section. The center section is made in two parts and contains channels through which liquid helium flows to cool the rubies. ${ }^{8}$ This was an attempt to improve the cooling, since some experiments had indicated that heating of the rubies may occur. This construction effectively eliminated all pressed contact thermal resistance between a number of metal-to-metal joints. These joints were located between the rubies and the main CCR 4.5-K station. Although there was a gain improvement of a few decibels, the improvement was not as large as had been hoped.

\footnotetext{
${ }^{7}$ T. Otoshi and R. C. Clauss, private communication.

${ }^{8}$ The two parts were vacuum furnace brazed by Scarrott Metallurgical, Los Angeles, California.
}

The ruby bars are firmly pushed against this center plate by spring-loaded copper pins. The rubies are flush at the pump end of the structure and tapered in the E-plane at the signal end of the structure. The tapered ruby sections are positioned in a six-step quarter-wave transformer to effect the transition from the 0.306-inch-wide waveguide of the circulator block to the 0.100 -inch-wide ruby-filled waveguide.

One potentially serious problem with this design is the leakage of signal power from the waveguide in the top section to the waveguide in the bottom section at both ends of the structure. To reduce this problem, we have found it necessary to lap the structure completely assembled (minus the rubies) with a special fixture. After lapping, the structure is never disassembled completely. The rubies are installed without disassembly of the structure.

\section{E. Circulator Block}

The circulator block consists of 18 symmetrical Y-junction $\mathrm{H}$-plane circulators. The circulators are also constructed in reduced-height waveguide. Each junction consists of two ferrite disks attached to the upper and lower waveguide broad walls. The disks are approximately 0.107 inch in diameter and 0.020 inch tall. The ferrite material used is type TT $2.111 .^{9}$ Each junction also employs a Teflon matching element, a departure from the small alumina matching elements used in past designs. This circulator design is basically a frequency. scaled version of a design used at $22 \mathrm{GHz}$ [4] .

The circulator block construction is similar to that of the maser block (see Fig. 8). It consists of a sandwich of three pieces of copper. Nine of the circulators are between the top two layers and nine are between the bottom two layers. The top and bottom rows are connected in series by an external length of waveguide.

\section{F. Superconducting Magnets}

There are two separate superconducting magnets in this maser. Both magnets operate in a vacuum and are cooled by thermal conduction to the liquid helium within the 4.5-K CCR station. The main magnet is used to provide the 11.8-kilogauss $\mathrm{dc}$ field required to Zeeman-split the energy levels to achieve a $32-\mathrm{GHz}$ frequency separation between levels 2 and 3 . The second magnet is used to bias the ferrite disks in the circulator block assembly. First, the magnet for biasing the rubies will be discussed (see Fig. 9).

The magnetic field is created by a single superconducting racetrack coil completely enclosed in a Hiperco (iron/cobalt

\footnotetext{
${ }^{9}$ Trans-Tech, Inc., Gaithersburg, Maryland. The material is a nickelzinc ferrite with a saturation magnetization near 7000 gauss at $4.2 \mathrm{~K}$.
} 
alloy) box. ${ }^{10}$ This magnet does not suffer from the fringing field problems associated with the open Cioffi-style magnets [7] used at K-band. The magnet coil form is rectangular with rounded corners and has inside dimensions of 7.2 by 3.57 inches. The coil form is 1.1 inches high and is constructed of copper. The magnet windings consist of 3056 turns of niobium-titanium (T48B) wire. ${ }^{11}$ There are approximately 40 layers with 76 turns per layer. The coil windings are prevented from moving by vacuum-impregnating the coil with Scotchcast 235 epoxy. ${ }^{12}$

The top and bottom plates of the Hiperco are 9.6 inches by 6.0 inches by 0.64 inch. They are each constructed of two separate pieces. Slots are machined for the signal waveguides in one plate and for the pump waveguides in the opposite plate. The side pieces are 1.030 inch high, approximately 0.640 inch wide, and of varying lengths.

The magnetic field taper is achieved by inserting a tapered Hiperco shim between the top plate and the sides. There is also 0.016 inch of steel shim over the four center rubies. This corrects a lateral field gradient of approximately 180 gauss between the inner and outer rubies. The current required to achieve 11.8 kilogauss is 12.6 amps. The magnet is operated in the persistent mode. The thermal switch consists of a small light bulb illuminating a small section of the superconducting wire where the copper cladding has been etched away.

The magnet for the circulators is much smaller and constructed differently (see Fig. 10). The Hiperco yoke is C-shaped with additional cylindrical pole pieces to get as much Hiperco as close to the ferrite as possible. The superconducting wire ${ }^{13}$ is wound on the Kapton-covered Hiperco. To bias the ferrites correctly at $4.5 \mathrm{~K}$, the coil is usually charged with 7.5 amps. It is also operated in the persistent mode.

\section{G. Microwave Pumps}

The question of how best to pump the ruby to obtain population inversion depends to some extent on the pump power required. It is well known that ruby becomes more difficult to pump at frequencies that are large compared to the zero field

\footnotetext{
${ }^{10}$ Carpenter Steel, Reading, Pennsylvania.

${ }^{11}$ Supercon, Inc., Shrewsbury, Massachusetts. The wire is a single filament with a superconducting core diameter of 0.005 inch with 0.0025 -inch copper cladding. The superconducting transition temperature is $9.5 \mathrm{~K}$.

${ }^{12} 3 \mathrm{M}$ Corporation, Saint Paul, Minnesota. The epoxy was added by the JPL cable shop. ${ }^{13}$ Also T48B wire with a 0.005 -inch niobium-titanium core and
0.0015 -inch copper cladding.
}

splitting of $11.5 \mathrm{GHz}$. It is rather easy to estimate the minimum pump power required if one assumes a relaxation rate for the pump transitions. One can use

$$
P_{\min }=h f_{p} w \alpha\left(\frac{N}{V}\right) V
$$

where

$$
\begin{aligned}
f_{p} & =\text { pump frequency } \\
w & =\text { relaxation rate } \\
N / V= & \text { spin density } \\
V= & \text { volume of maser material } \\
\alpha= & \text { a constant which depends on the population distribu- } \\
& \text { tion because all the spins need not be flipped }
\end{aligned}
$$

In this case, $f_{p}=66.4 \mathrm{GHz}, \alpha=0.17, V=2.29 \mathrm{cc}, w$ is estimated to be $100 / \mathrm{sec}$, and $N / V=2.35 \mathrm{E} 19 / \mathrm{cc}$. Therefore, $P_{\min }=40 \mathrm{~mW}$. However, Eq. (2) is a lower bound on the required pump power. It underestimates the required power for several reasons. First, it assumes that every photon incident on the ruby is successful in flipping a spin. This is a very poor assumption for the specific ruby operating point we are using because of the low transition probabilities. It is a particularly bad assumption when the ruby is located in an electromagnetic structure with a low $Q$ at the pump frequency. Second, it ignores the fact that the pump RF magnetic field may not be properly polarized. Third, it ignores waveguide losses and losses due to mismatch.

In addition to the estimates, some experiments were carried out using a single bar of the ruby in liquid helium. We found that approximately $40 \mathrm{~mW}$ was required at the input to a single ruby channel in order to achieve an inversion ratio near 1.0 over $500 \mathrm{MHz}$ of signal bandwidth. After adding in the waveguide losses and window losses, we estimated that each stage would require at least $50 \mathrm{~mW}$. Therefore, the total requirement for eight stages would be $400 \mathrm{~mW}$. Because of the large power requirement, it was decided to use four IMPATT diode oscillators, each supplying approximately $100 \mathrm{~mW}$ of $\mathrm{CW}$ power. ${ }^{14}$ The method by which the pump power was directed to the rubies was described earlier.

The coupling of the pump power to the rubies was accomplished with quarter-wave matching elements. The design of the quarter-wave matching elements was based on a wave impedance given by

\footnotetext{
${ }^{14}$ Model 47134H, Hughes Aircraft Company, Electron Dynamics Division, Torrance, California.
} 


$$
Z=\frac{b}{a} \frac{\lambda_{g}}{\lambda_{0}}
$$

Then we use the matching condition for quarter-wave transformers, i.e., $Z$ (matching section) $=Z_{m}$ :

$$
Z_{m}=\sqrt{Z_{1} \cdot Z_{2}}
$$

where $Z_{1}=$ impedance of the dielectric-filled guide and $Z_{2}=$ impedance of WR15. Then $Z_{m}$ is given by

$$
Z_{m}=\sqrt{\frac{b_{1} b_{2}}{a_{1} a_{2}}} \frac{\sqrt{\lambda_{g}^{(1)} \lambda_{g}^{(2)}}}{\lambda_{0}}=\sqrt{\frac{b_{m}}{a_{m}}} \frac{\lambda_{g}^{(m)}}{\lambda_{0}}
$$

Thus $b_{m}=0.061$ and $a_{m}=0.122 . \epsilon_{m}$ is chosen so that

$$
\lambda_{g}^{(m)}=\sqrt{\lambda_{g}^{(1)} \lambda_{g}^{(2)}}=\frac{\lambda_{0}}{\sqrt{\epsilon_{m}-\left(\lambda / \lambda_{c 0}\right)^{2}}}
$$

This requires that $\epsilon_{m}=2.9$. We used a dielectric (polystyrene doped with titanium) with a dielectric constant $\epsilon$ of 3 . The thickness of the elements is 0.029 inch. Experimentally, the return loss looking into the pump end of a ruby bar with the matching element was approximately $15 \mathrm{~dB}$. The IMPATT diode oscillators are frequency modulated with a bandwidth roughly twice the desired signal bandwidth. The modulation rate is typically $4 \mathrm{kHz}$.

\section{H. Cryogenics}

A closed-cycle helium refrigerator (CCR) is used to provide a 4.5-K environment for the maser (see Fig. 11). This refrigerator uses a CTI-Cryogenics Model 1020 drive unit to precool a Joule-Thomson expansion circuit. The refrigerator is capable of more than 3 watts of cooling at $4.5 \mathrm{~K}$. In addition, we have installed an externally adjustable Joule-Thomson valve that permits operating at reduced helium flow rates. This allows us to achieve temperatures lower than $4.5 \mathrm{~K}$ by reducing the vapor pressure over the helium with a vacuum pump. ${ }^{15}$ This produces an additional $10 \mathrm{~dB}$ of gain at the expense of reserve cooling capacity.

\footnotetext{
${ }^{15}$ A Leybold-Heraeus model S65B vacuum pump that has a pumping speed of 45.9 cubic feet per minute was used. This model pump will be used at OVRO.
}

\section{Maser Package}

The entire maser and refrigerator assembly (minus the drive unit) is contained within a cylindrical stainless steel vacuum housing approximately 12.5 inches in its inside diameter (see Fig. 12). The four IMPATT diode oscillators are mounted on a temperature-controlled copper plate attached to the vacuum housing.

The refrigerator and maser are both surrounded by a cylindrical copper radiation shield near $70 \mathrm{~K}$, and the maser is further shielded by a cylindrical copper radiation shield near $5 \mathrm{~K}$. The maser package weighs approximately 200 pounds.

For mounting in the antenna, the maser, the receiver, and various pieces of support equipment are housed in a circular container 18.75 inches in diameter and 84 inches long. The estimated total weight is approximately 400 pounds.

\section{Performance}

The performance of the maser with the field tapered as described in Section IIA yielded a net gain of $21 \mathrm{~dB}$ and an instantaneous bandwidth of $400 \mathrm{MHz}$ at $4.5 \mathrm{~K}$ (see Fig. 5). This is the maximum gain-bandwidth product we have achieved. Higher gains with reduced bandwidth can be obtained by decreasing the frequency modulation bandwidth of the "pump" oscillators and reducing the magnetic field gradient. The amount of ruby absorption is difficult to measure with our measurement setup (all components at $32 \mathrm{GHz}$ ). The power absorption is so large (because of the large volume of ruby) that the power level at the output of the maser is below the sensitivity of our diode detectors. However, the absorption certainly exceeds $36 \mathrm{~dB}$. Therefore, the inversion ratio (gain with pumps on/absorption with pumps off) is less than 1.0 and probably closer to 0.7 . The ruby is considered to be saturated when an inversion ratio of approximately 1.1 is achieved [8] .

The noise temperature was measured as a function of gain by measuring output power with reference terminations at different temperatures connected to the maser. In this case, the "hot load" was an ambient target placed over the horn and the "cold load" was the sky. The maser output was downconverted to S-band with a superheterodyne mixer. Commercial S-band amplifiers, a tunable filter, and a power meter enabled us to compare the maser output power when the input was looking at the hot and cold loads. The measured maser noise temperatures (including the feedhorn) were as follows (see Fig. 6):

$$
\begin{aligned}
& \text { Net gain of } 40 \mathrm{~dB}: T=17 \pm 2.5 \mathrm{~K} \\
& \text { Net gain of } 30 \mathrm{~dB}: T=20 \pm 2.5 \mathrm{~K}
\end{aligned}
$$


Net gain of $20 \mathrm{~dB}: T=25 \pm 2.5 \mathrm{~K}$

The error was estimated from the scatter in the measured values. Since these results were all obtained with a fixed mag. netic field taper, in principle the noise temperature obtained with $40 \mathrm{~dB}$ of gain could be realized across a full $400-\mathrm{MHz}$ bandwidth if there were sufficient pump power and if ruby heating was not a problem. The gain ripple can be as low as $\pm 1 \mathrm{~dB}$ with adjustment of the magnetic field, IMPATT center frequencies, and sweep bandwidths.

\section{Applications}

This wide-bandwidth maser will find immediate application in continuum radio astronomy as well as in deep space spacecraft communications. In addition to using this maser to search for spatial anisotropies in the cosmic background, other potential experiments include tracking the fourth harmonic of the Voyager or Galileo spacecraft X-band signal to study weather effects on a deep space communications link at $33.6 \mathrm{GHz}$.

\section{Acknowledgments}

The authors would like to thank several of their colleagues at JPL for very useful discussions. In particular, thanks are due to Bob Clauss, Sam Petty, and Javier Bautista for matters relating to the maser construction and testing. Also, thanks to Dan Hoppe for his assistance and for the use of his computer program for analyzing the pump matching as well as to Tom Otoshi for discussions relating to the VSWR measurements. Thanks also to Dr. Charles Lawrence of the Caltech Astronomy Department for his assistance in the final finishing stages of the maser and its installation at the Owens Valley Radio Observatory. 


\section{References}

[1] R. M. Dickinson, A Comparison of 8.415, 32.0, and 565646-GHz Deep Space Telemetry Links, JPL Publication 85-71, Jet Propulsion Laboratory, Pasadena, California, 1985.

[2] J. G. Smith, "Ka-Band (32-GHz) Downlink Capability for Deep Space Communications," TDA Progress Report 42-88, vol. October-December 1986, Jet Propulsion Laboratory, Pasadena, California, pp. 96-103, February 15, 1987.

[3] N. F. deGroot, "Ka-Band (32-GHz) Allocations for Deep Space," TDA Progress Report 42-88, vol. October-December 1986, Jet Propulsion Laboratory, Pasadena, California, pp. 104-109, February 15, 1987.

[4] C. R. Moore and R. C. Clauss, "A Reflected-Wave Ruby Maser with K-Band Tuning Range and Large Instantaneous Bandwidth," IEEE Trans., vol. MTT-27, pp. 249$256,1979$.

[5] A. E. Siegman, Microwave Solid State Masers, New York: McGraw.Hill, 1964.

[6] W. H. Higa, "Noise Performance of Traveling-Wave Masers," IEEE Trans., vol. MTT-12, p. 139, 1964.

[7] P. P. Cioffi, "Approach to the Ideal Magnetic Circuit Concept Through Superconductivity," J. Appl. Phys., vol. 33, pp. 875-879, March 1962.

[8] C. R. Moore and D. Neff, "Experimental Evaluation of a Ruby Maser at $43 \mathrm{GHz}$," IEEE Trans., MTT-30, pp. 2013-2015, 1982. 
Table 1. Estimated noise temperature contributions (in kelvins) from various components of the maser/receiver for three different values of net gain

\begin{tabular}{lccc}
\hline & Variable/stage & \multicolumn{3}{c}{ Net gain, dB } \\
\cline { 2 - 4 } & 40 & 30 & 20 \\
\hline 1 & 5.4 & 6.0 & 6.8 \\
2 & 1.8 & 2.6 & 3.9 \\
3 & 0.5 & 1.1 & 2.2 \\
4 & 0.2 & 0.5 & 1.2 \\
5 & 0.1 & 0.2 & 0.7 \\
6 & - & 0.1 & 0.4 \\
7 & - & - & 0.2 \\
8 & - & - & 0.1 \\
Zero point energy & 0.8 & 0.8 & 0.8 \\
$T_{\text {equiv }}$ (4.5-K input) & 8.8 & 11.3 & 16.3 \\
Waveguide inputs (estimated) & 3 & 3 & 3 \\
$T_{\text {equiv }}$ (ambient) & 11.8 & 14.3 & 19.3 \\
Feedhorn (estimated) & 4 & 4 & 4 \\
$T_{\text {total }}$ & 15.8 & 18.3 & 23.3 \\
\hline
\end{tabular}




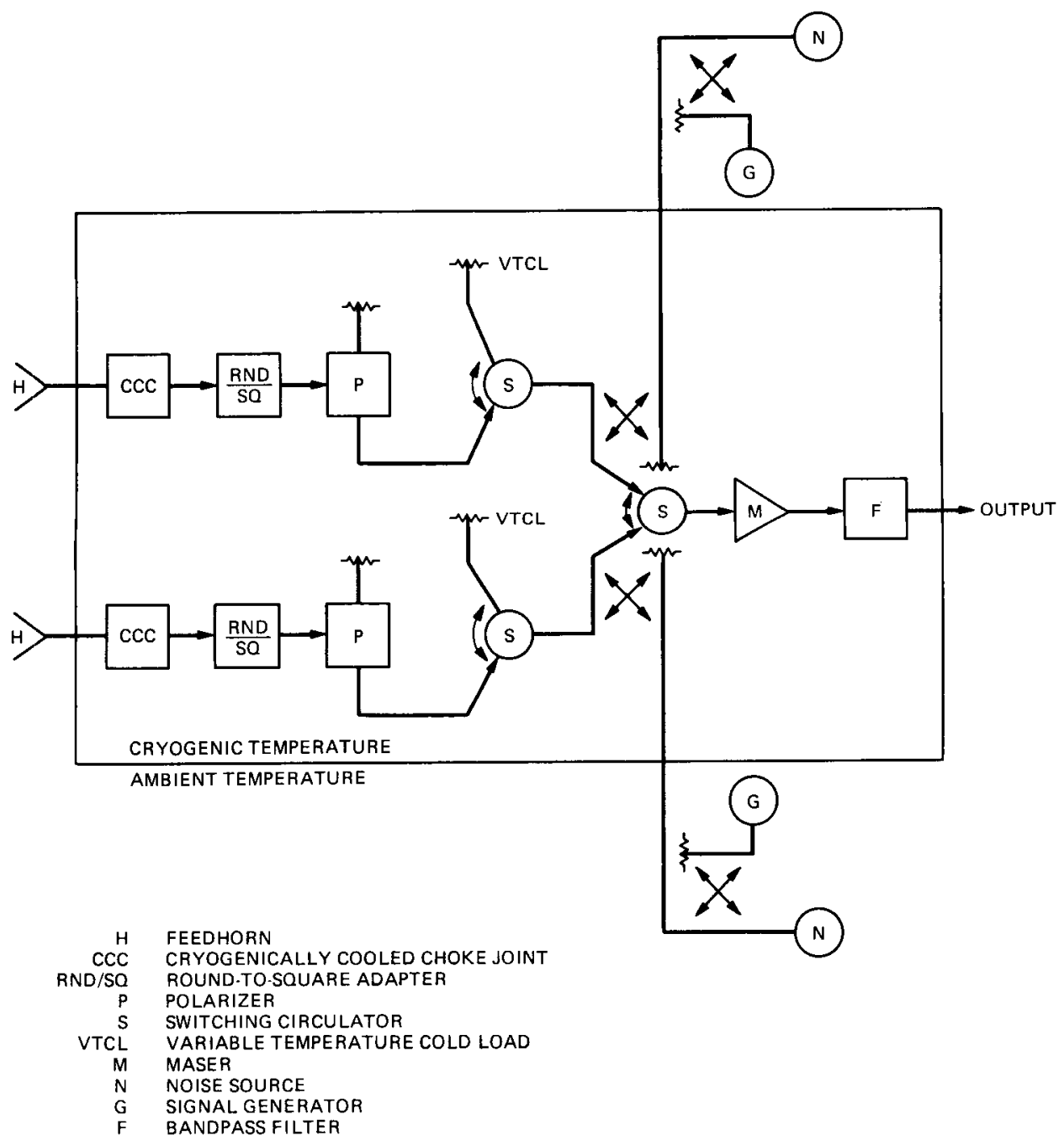

Fig. 1. Receiver input block diagram 


\section{ORIGINAL PAGE IS \\ OF POOR QUALITY}

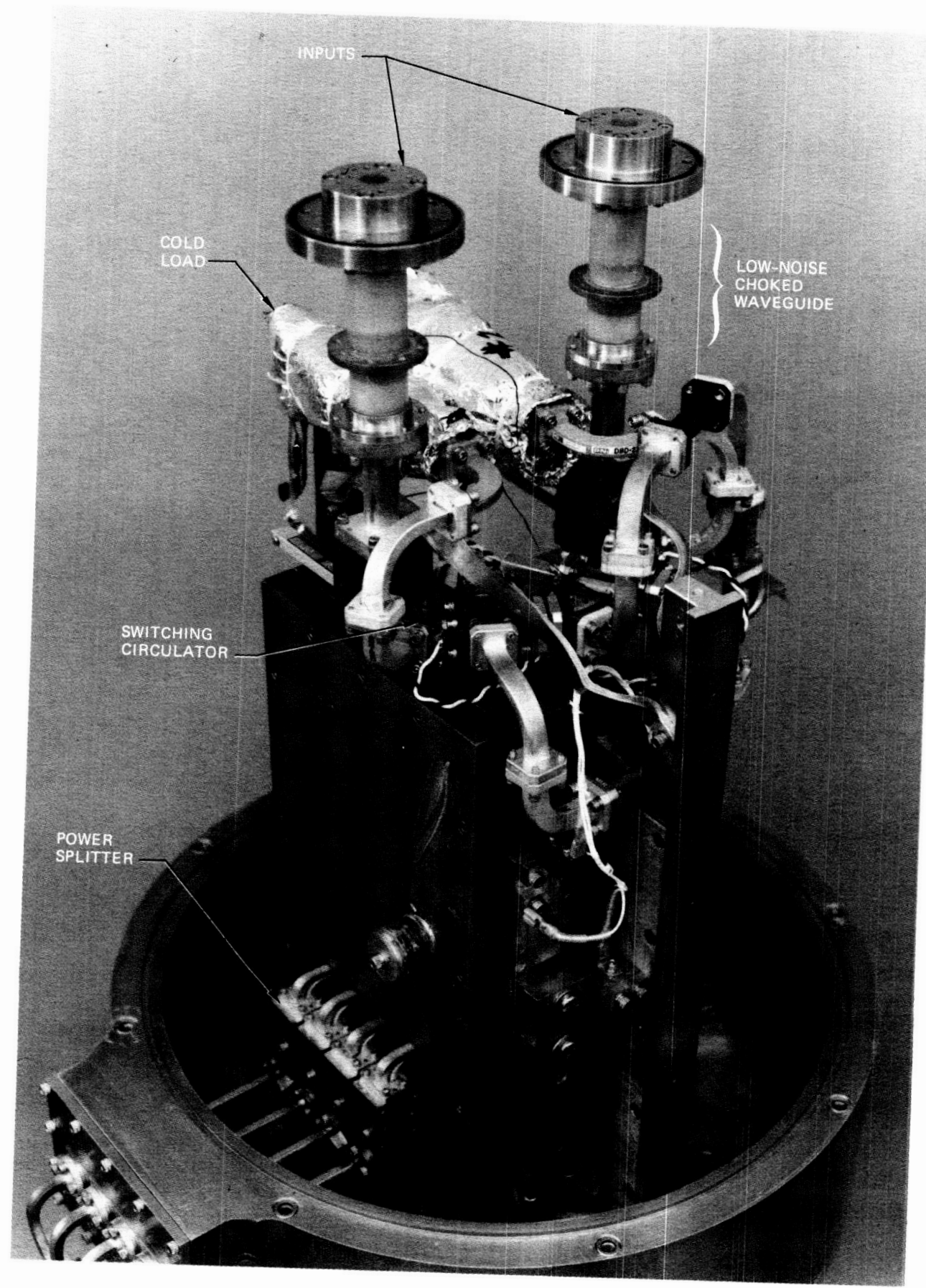

Fig. 2. View of the input feeds, waveguide plumbing, and maser 


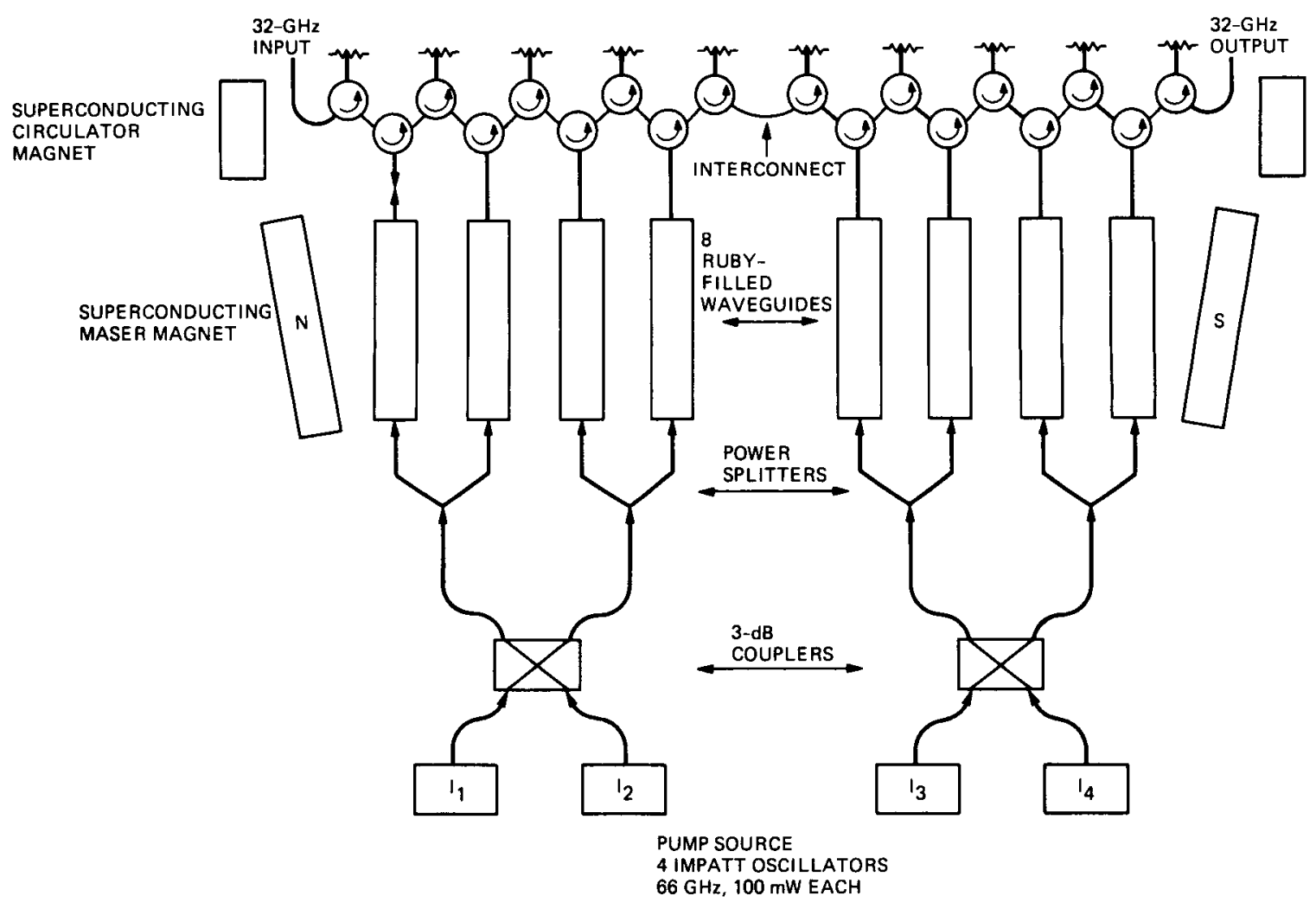

Fig. 3. 32-GHz reflectod-wave maser block diagram 


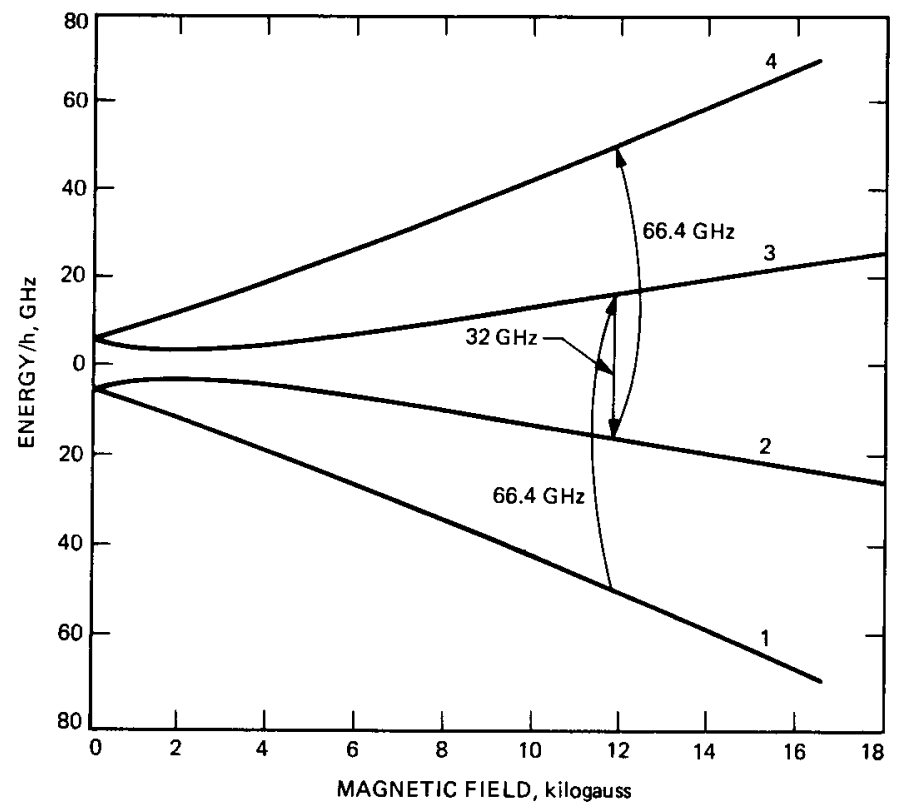

Fig. 4. Ground state energy levels of ruby with optic-axis orientation of 54 degrees 44 minutes. Upward pointing arrows denote pump transitions and downward pointing arrow denotes signal transition.

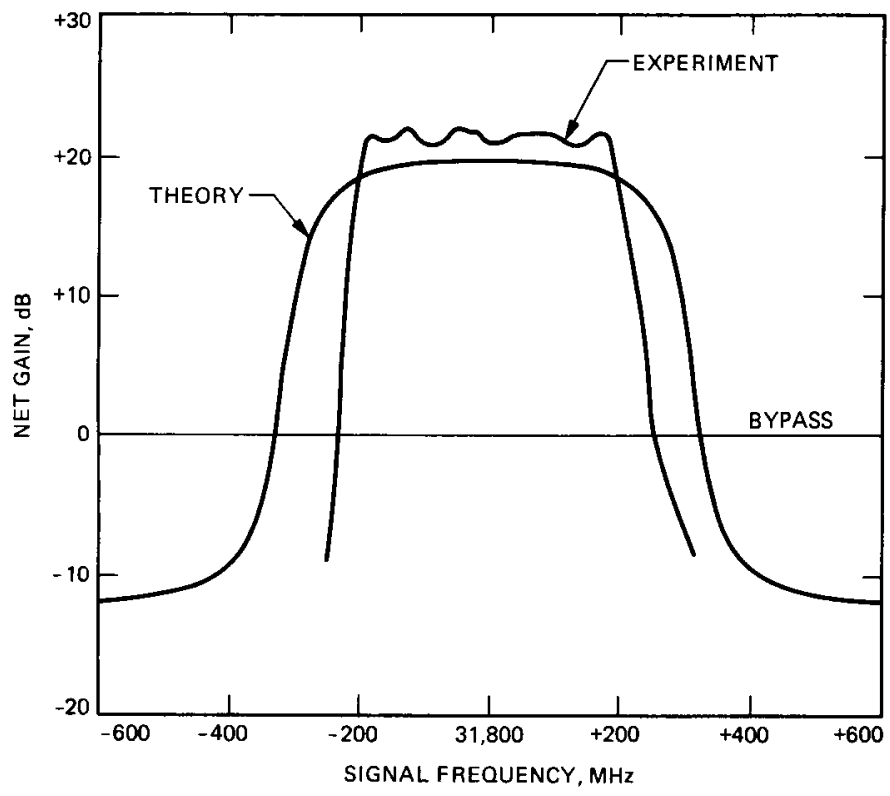

Fig. 5. Theoretical bandpass using equation (1) with $g=232 \mathrm{~dB}$ and $d v=622 \mathrm{MHz}$ superimposed on the experimentally measured bandpass

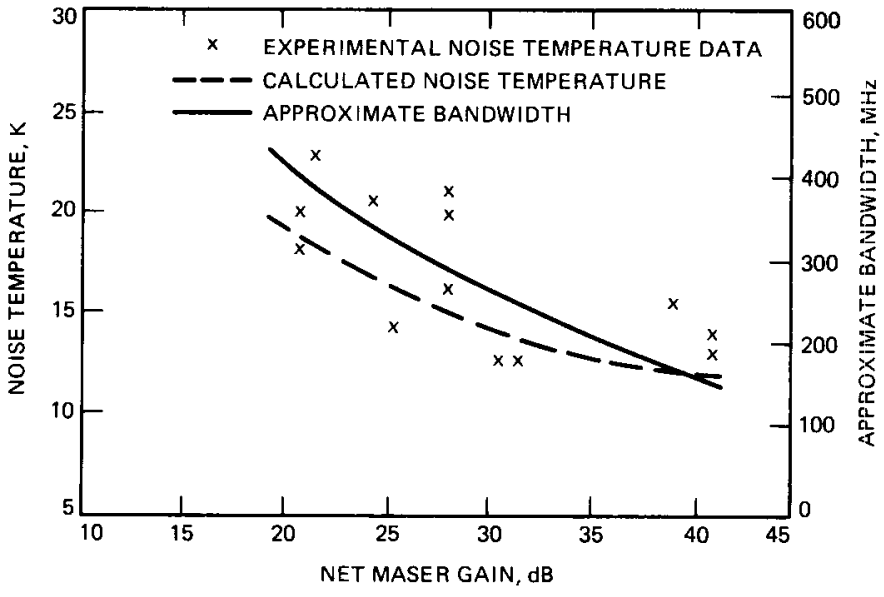

Fig. 6. Experimental and estimated noise temperatures as a function of maser gain and bandwidth 


\section{ORIGINAL PAGE IS \\ OF POOR QUALITY}

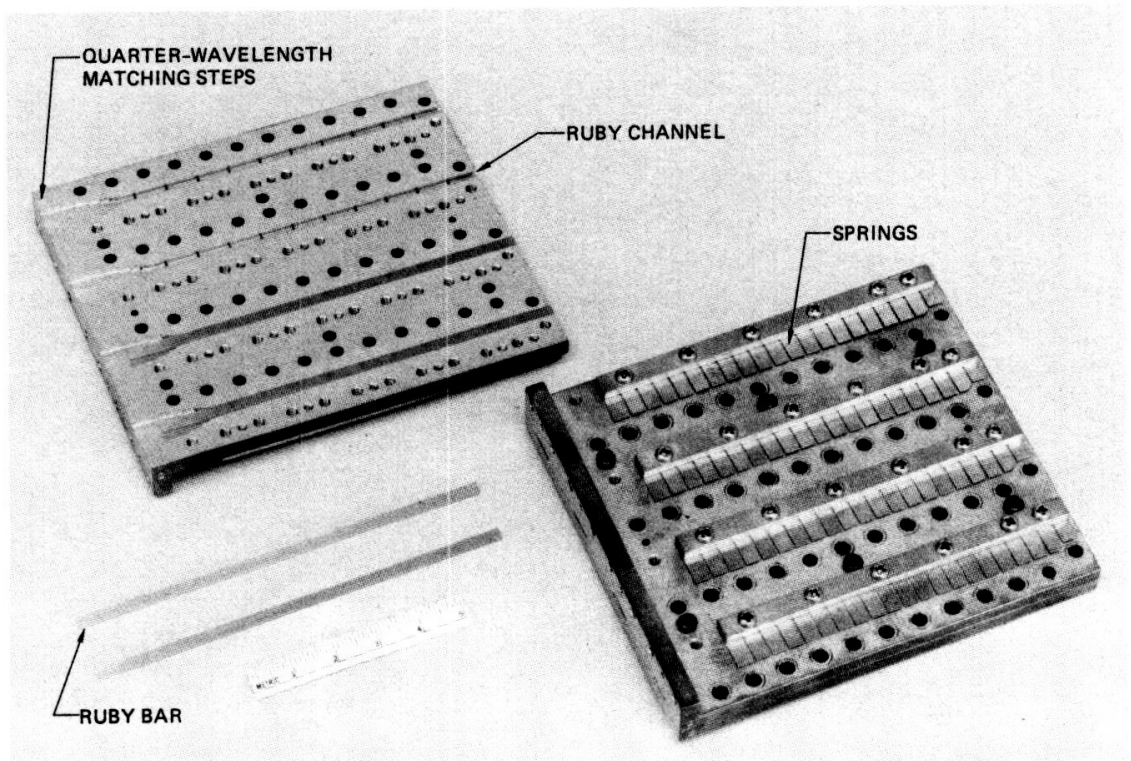

Fig. 7. Partially disassembled view of the ruby-filled structure. The helium lines to the center dividing plate are not shown.

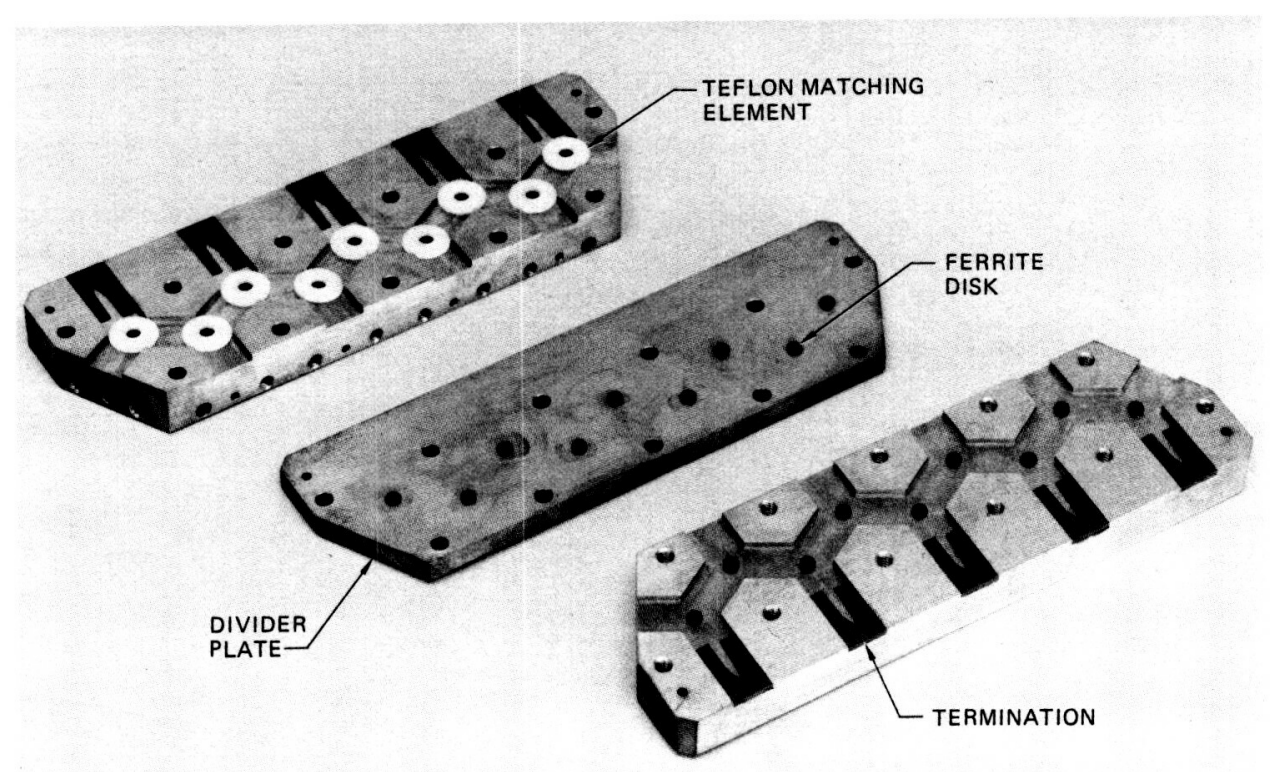

Fig. 8. Disassembled view of the circulator block 


\section{ORIGINAL PAGE IS \\ OF POOR QUALITY}

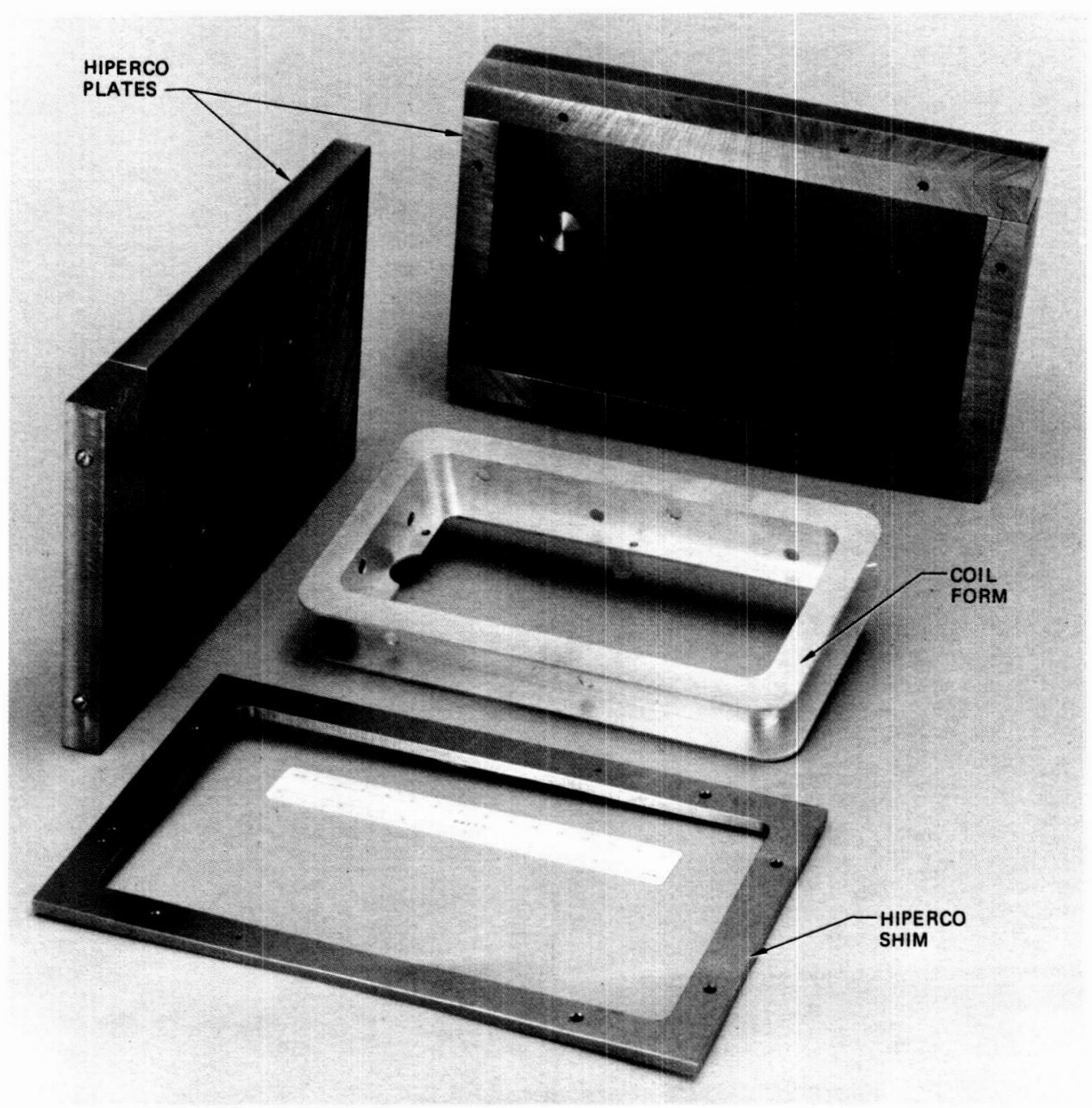

Fig. 9. View of the Hiperco box and copper coil form before the winding of the superconducting coil. The holes for the pump waveguide are not shown. 


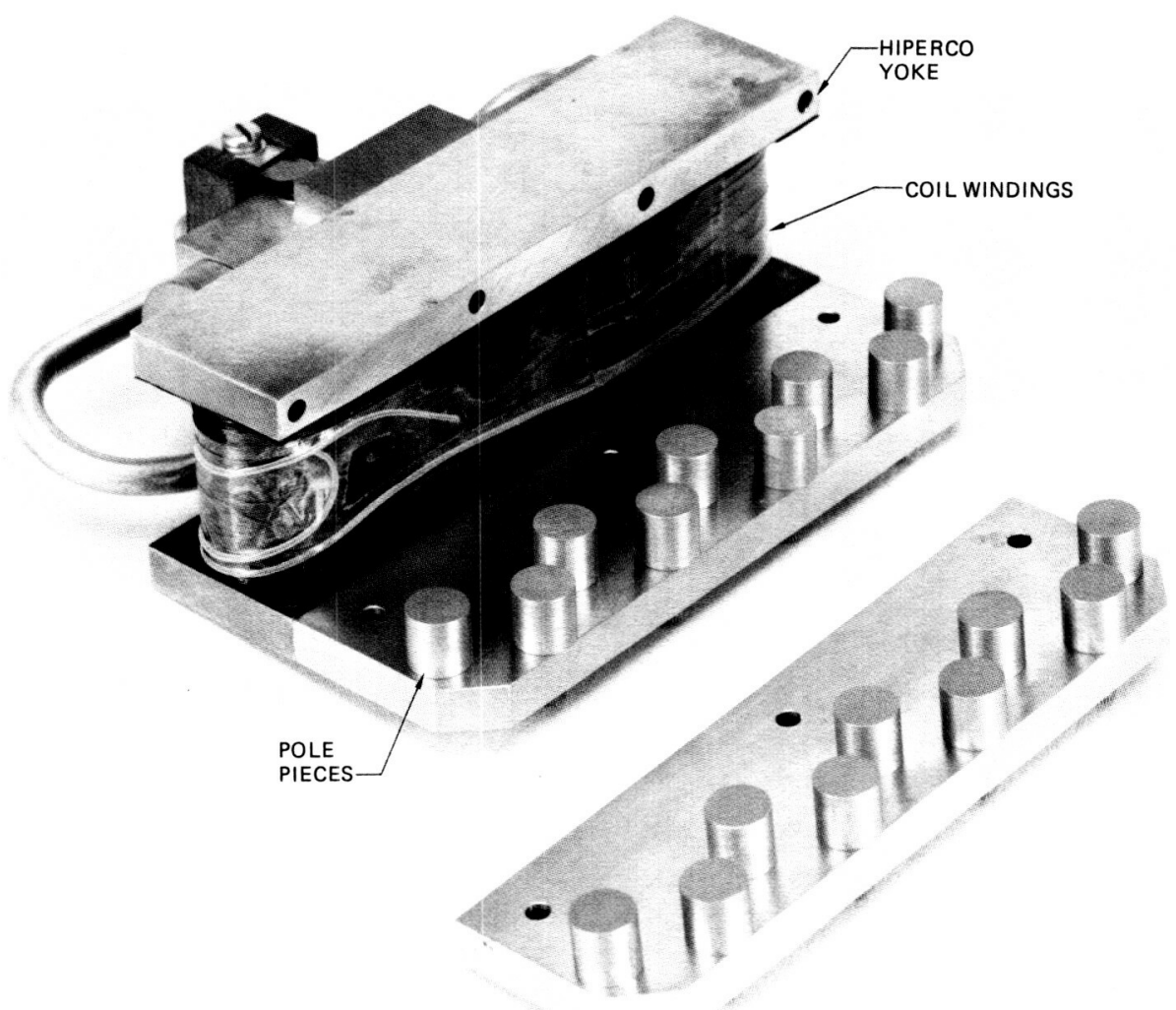

Fig. 10. Partially disassembled view of the circulator electromagnet

ORIGINAL PAGE IS OF POOR QUALITY 
ORICUNA PACE IS

OF POOR QUALITY

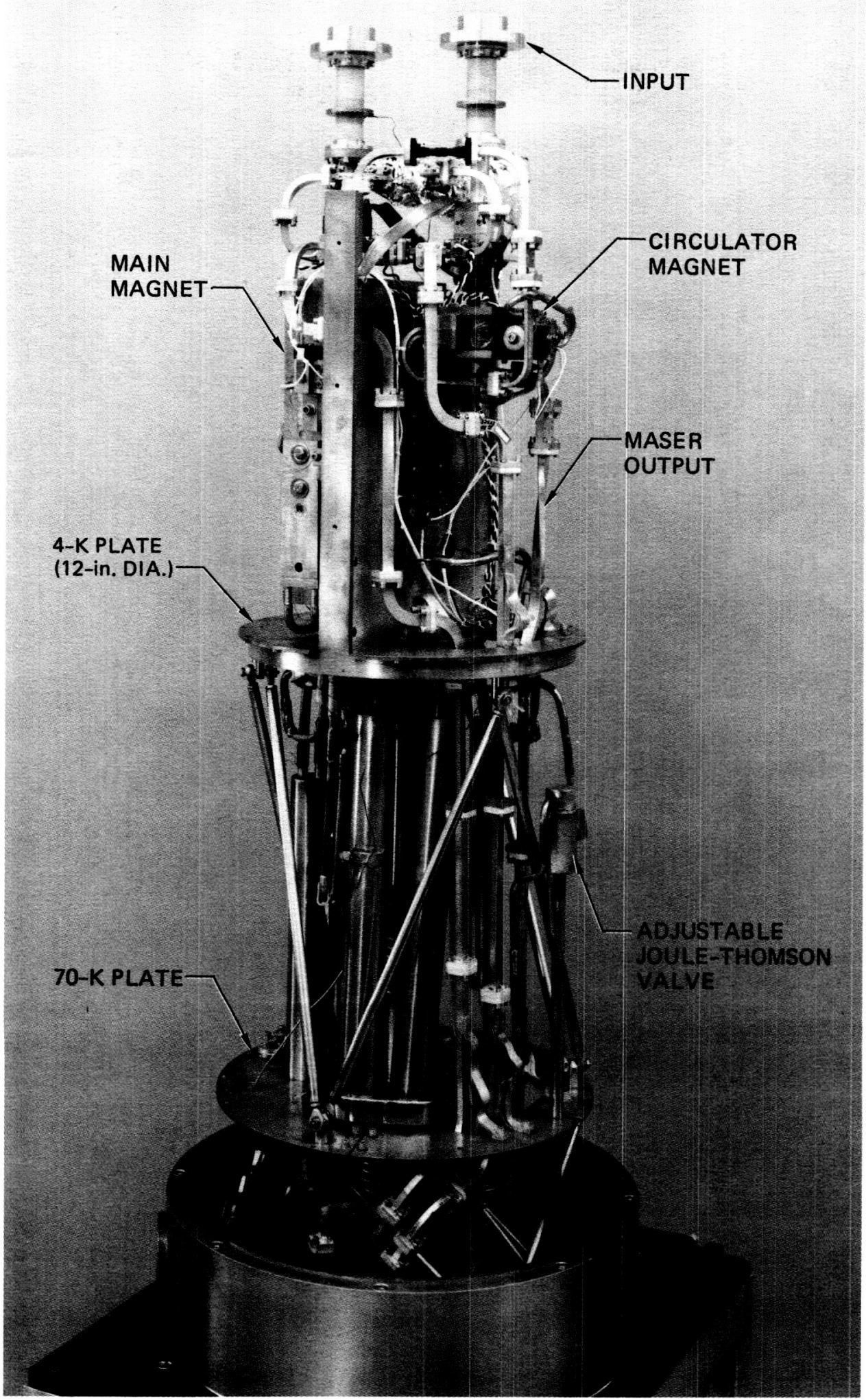

Fig. 11. Rear overview of the complete maser. The radiation shields and vacuum housings have been removed. 


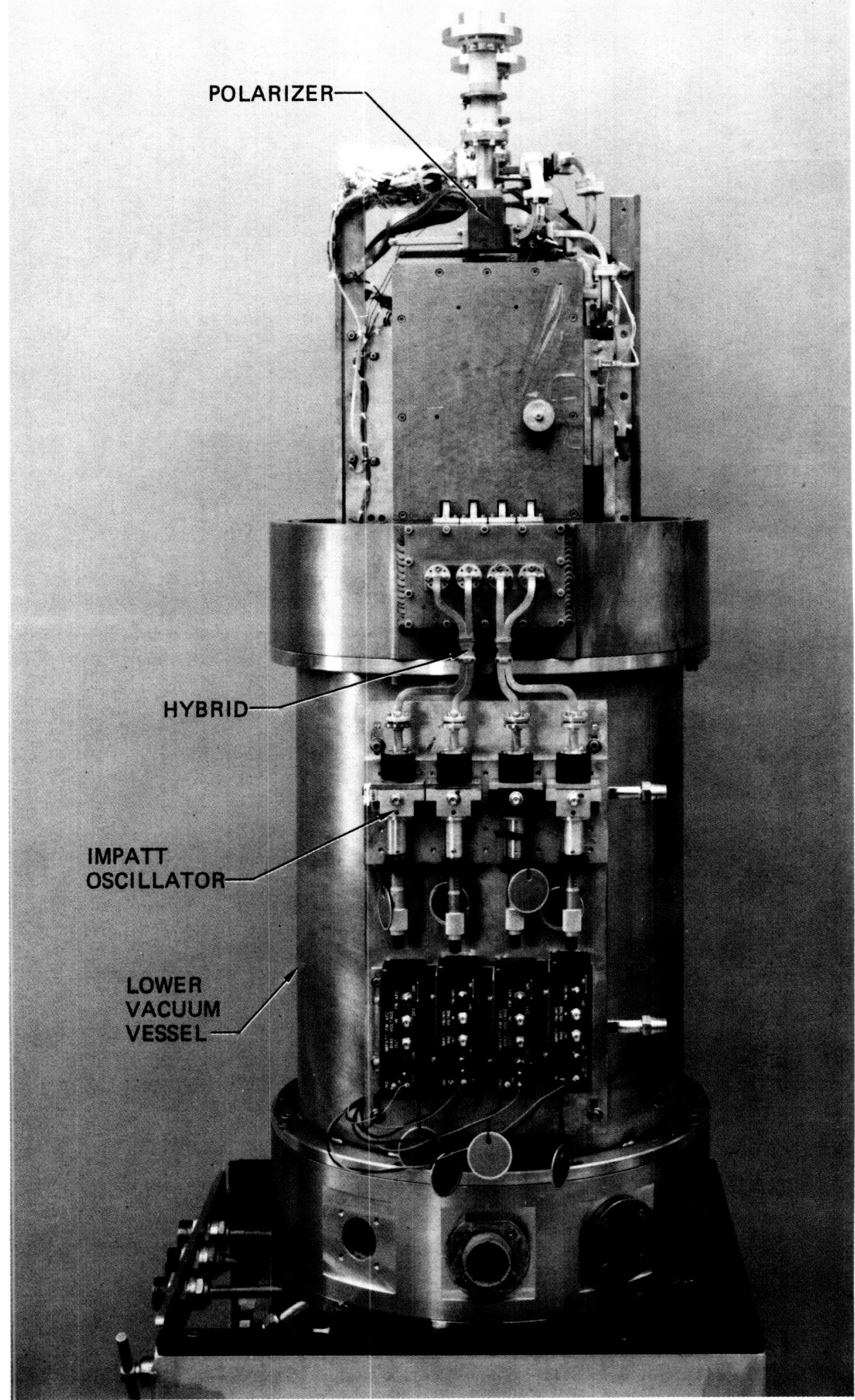

Fig. 12. Front overview of the complete maser. The upper vacuum housing and radiation shields have been removed. 